\title{
Spectral scalability and optical spectra of fractal multilayer structures: FDTD analysis
}

\author{
Sevket Simsek $^{1} \cdot$ Selami Palaz $^{2} \cdot$ Amirullah M. Mamedov ${ }^{3,4} \cdot$ Ekmel Ozbay $^{3}$
}

Received: 3 August 2016/Accepted: 3 December 2016/Published online: 19 December 2016

(C) Springer-Verlag Berlin Heidelberg 2016

\begin{abstract}
An investigation of the optical properties and band structures for the conventional and Fibonacci photonic crystals (PCs) based on $\mathrm{SrTiO}_{3}$ and $\mathrm{Sb}_{2} \mathrm{Te}_{3}$ is made in the present research. Here, we use one-dimensional $\mathrm{SrTiO}_{3}-$ and $\mathrm{Sb}_{2} \mathrm{Te}_{3}$-based layers. We have theoretically calculated the photonic band structure and transmission spectra of $\mathrm{SrTiO}_{3}$ - and $\mathrm{Sb}_{2} \mathrm{Te}_{3}$-based $\mathrm{PC}$ superlattices. The position of minima in the transmission spectrum correlates with the gaps obtained in the calculation. The intensity of the transmission depths is more intense in the case of higher refractive index contrast between the layers.
\end{abstract}

\section{Introduction}

Photonic crystals (PhCs) are structured dielectric composites that are designed and fabricated to have periodic optical properties that strongly alter the properties and propagation of light. One of the defining properties of $\mathrm{PhCs}$ is photonic band gaps-frequency ranges where light

Amirullah M. Mamedov

mamedov@bilkent.edu.tr

1 Department of Material Science and Engineering, Hakkari University, Hakkari, Turkey

2 Faculty of Science and Letters, Harran University, Sanliurfa, Turkey

3 Nanotechnology Research Center, Bilkent University, Ankara, Turkey

4 International Scientific Center, Baku State University, Baku, Azerbaijan cannot propagate because of destructive interference between coherent scattering paths. The same situation we can observe in the PhCs-based superlattices [1, 2]. The structures intermediate between the periodic and disordered structures (quasiperiodic structure)-the Fibonacci and Thue-Morse superlattices, occupy a special place among the superlattices. The strong resonances in spectral dependences of fractal multilayers can localize light very effectively. In addition, long-range ordered aperiodic photonic structures offer an extensive flexibility for the design of optimized light-emitting devices, and the theoretical understanding of the complex mechanisms governing optical gaps and mode formation in aperiodic structures becomes increasingly more important. The formation of photonic band gaps and the existence of quasi-localized light states have already been demonstrated for one (1D)and two-dimensional (2D) aperiodic structures based on Fibonacci and the Thue-Morse sequences [2]. The unusual electron properties of quasiperiodic potentials have also stimulated extensive research of the optical counterparts. However, to the best of our knowledge, a rigorous investigation of the band gaps and optical properties in the more complex types of aperiodic structures has not been reported so far.

In this paper, we investigated the energy spectrum and optical properties in the Fibonacci-type photonic band gap (PBG) structures consisting of nonlinear material $\left(\mathrm{SrTiO}_{3}\right.$ and $\mathrm{Sb}_{2} \mathrm{Te}_{3}$ ) [3] in detail by using the finite-difference time-domain (FDTD) method and the plane wave expansion method (PWE). The choice of the $\mathrm{SrTiO}_{3}$ and $\mathrm{Sb}_{2} \mathrm{Te}_{3}$ crystals as the active media for our investigation was associated with their unusual optical properties. It is well known that $\mathrm{SrTiO}_{3}$ and $\mathrm{Sb}_{2} \mathrm{Te}_{3}$ are the virtual ferroelectric material, and their properties are very sensitive to external influences (temperature, electric field, stress and light). 


\section{Computational details}

\subsection{Fibonacci sequences and model}

Quasiperiodic structures are nonperiodic structures that are constructed by a simple deterministic generation rule. A quasiperiodic system occurs when two or more incommensurate periods are superimposed. Therefore, it is neither aperiodic nor a random system and can be considered as intermediate the two [1]. In other words, due to a long-range order a quasiperiodic system can form forbidden frequency regions called pseudoband gaps similar to the band gaps of a PC and simultaneously posses localized states as in disordered media [2]. The Fibonacci multilayer structure (well-known quasiperiodic structure) has been studied in past decade, and recently, the resonant states at the band edge of the photonic structure in the Fibonacci sequence are studied experimentally, too [3]. A 1D quasiperiodic Fibonacci sequence is based on a recursive relation, which has the form, $S_{j+1}=\left\{S_{j-1}, S_{j}\right\}$ for $j \gg 1$, with $S_{0}=\{B\}$, $S_{1}=\{\mathrm{A}\}, \quad S_{2}=\{\mathrm{BA}\}, \quad S_{3}=\{\mathrm{ABA}\}, \quad S_{4}=\{\mathrm{BAABA}\}$ and soon, where $S_{\mathrm{j}}$ is a structure obtained after $\mathrm{j}$ iterations of the generation rule [1]. Here, $A$ and $B$ are defined as being two dielectric materials, with different refractive indices $\left(n_{\mathrm{A}}, n_{\mathrm{B}}\right)$, and have geometrical layer thickness $\left(d_{\mathrm{A}}, d_{\mathrm{B}}\right)$. Materials $\mathrm{A}$ and $\mathrm{B}$ were considered as the conducting $\left(\mathrm{Sb}_{2} \mathrm{Te}_{3}\right.$ as topological insulator) material and dielectric material $\left(\mathrm{SrTiO}_{3}\right)$, respectively.

Figure 1a, b shows schematically the geometry of conventional photonic crystal (CPCs) and Fibonacci photonic crystal (FPCs). The thickness of the considered layers of $\mathrm{A}$ and $\mathrm{B}$ is $d_{\mathrm{A}}=0.5 \mathrm{a}$ and $d_{\mathrm{B}}=0.5 \mathrm{a}$, respectively. The lattice constant is $a=\left(d_{\mathrm{A}}+d_{\mathrm{B}}\right)=1 \mu \mathrm{m}$. The filling fraction $\mathrm{f}$ is the ratio between the thickness of

(a)

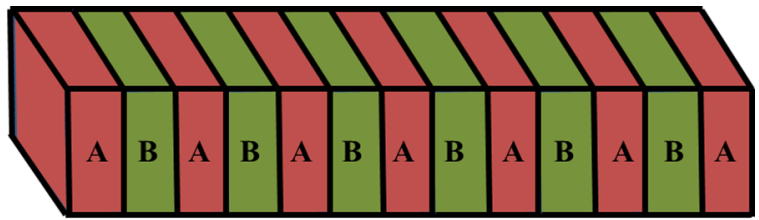

(b)

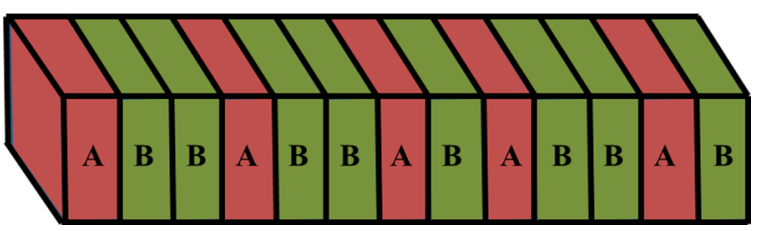

Fig. 1 1-Dimensional conventional photonic crystal structure (a) and Fibonacci photonic crystal structure (b)

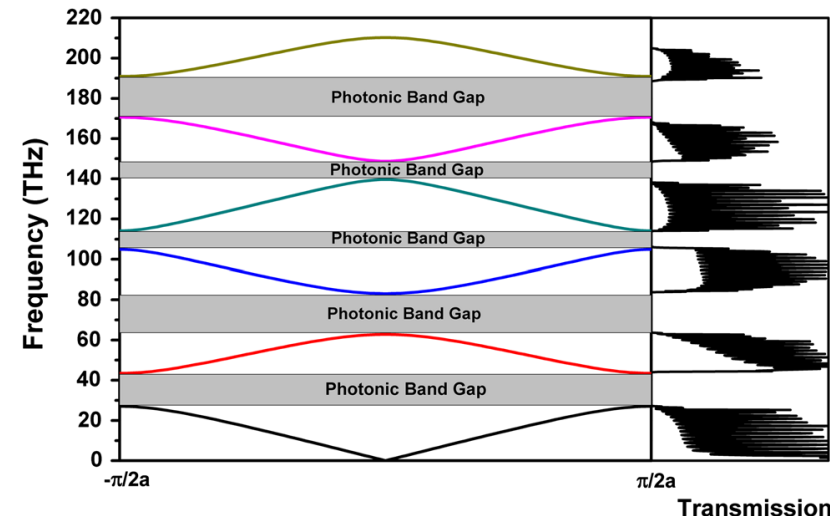

Fig. 2 TE band structure and transmittance spectra of $\mathrm{Sb}_{2} \mathrm{Te}_{3}$ and $\mathrm{SrTiO}_{3}$ compounds in 1D

Table 1 Variation of full band gap size for TE modes with filling factor for $\mathrm{Sb}_{2} \mathrm{Te}_{3}$ - and $\mathrm{SrTiO}_{3}$-based layers

\begin{tabular}{|c|c|c|c|c|c|c|}
\hline \multirow[t]{2}{*}{$\mathrm{FF}$} & \multicolumn{2}{|l|}{ TE1 } & \multicolumn{2}{|l|}{ TE2 } & \multicolumn{2}{|l|}{ TE3 } \\
\hline & BG & GS & BG & GS & BG & GS \\
\hline 0.1 & $(44-64)$ & 15.51 & $(96-127)$ & 13.90 & $(156-186)$ & 8.77 \\
\hline 0.2 & $(36-62)$ & 26.53 & $(90-109)$ & 9.54 & $(142-154)$ & 4.05 \\
\hline 0.3 & $(31-57)$ & 29.54 & $(87-89)$ & 1.13 & $(120-145)$ & 9.4 \\
\hline 0.4 & $(29-50)$ & 26.58 & $(72-88)$ & 10.00 & $(115-123)$ & 3.30 \\
\hline 0.5 & $(27-43)$ & 22.85 & $(63-83)$ & 13.69 & $(105-114)$ & 4.11 \\
\hline 0.6 & $(26-38)$ & 18.75 & $(57-75)$ & 13.63 & $(92-109)$ & 8.45 \\
\hline 0.7 & $(25-34)$ & 15.25 & $(53-67)$ & 11.66 & $(83-100)$ & 9.28 \\
\hline 0.8 & $(25-30)$ & 9.09 & $(51-61)$ & 8.92 & $(78-91)$ & 7.69 \\
\hline 0.9 & $(25-27)$ & 3.84 & $(50-55)$ & 4.76 & $(75-82)$ & 4.4 \\
\hline
\end{tabular}

\begin{tabular}{|c|c|c|c|c|}
\hline \multirow[t]{2}{*}{$\mathrm{FF}$} & \multicolumn{2}{|l|}{ TE4 } & \multicolumn{2}{|l|}{ TE5 } \\
\hline & BG & GS & BG & FF \\
\hline 0.1 & $(219-237)$ & 1.22 & $(280-283)$ & 0.53 \\
\hline 0.2 & $(184-212)$ & 5.28 & $(244-252)$ & 1.61 \\
\hline 0.3 & $(174-179)$ & 7.05 & $(208-233)$ & 5.66 \\
\hline 0.4 & $(148-171)$ & 6.49 & $(195-204)$ & 2.55 \\
\hline 0.5 & $(140-149)$ & 1.22 & $(171-191)$ & 5.52 \\
\hline 0.6 & $(130-139)$ & 7.07 & $(164-168)$ & 1.20 \\
\hline 0.7 & $(115-132)$ & 8.08 & $(149-162)$ & 4.18 \\
\hline 0.8 & $(106-121)$ & 7.04 & $(135-150)$ & 5.26 \\
\hline 0.9 & $(101-110)$ & 4.06 & $(127-137)$ & 3.78 \\
\hline
\end{tabular}

$F F$ filling factor, $B G$ band gap in $\mathrm{THz}, G S \%$ gap size

the lower refractive index layer (air) and the period of the PC, i.e., $f=d_{\mathrm{A}} /\left(d_{\mathrm{A}}+d_{\mathrm{B}}\right)$. The filling fraction is set to 0.5. The refractive index contrasts of $\mathrm{Sb}_{2} \mathrm{Te}_{3}$ and $\mathrm{SrTiO}_{3}$ are taken as in Refs [4] and [5], respectively. 

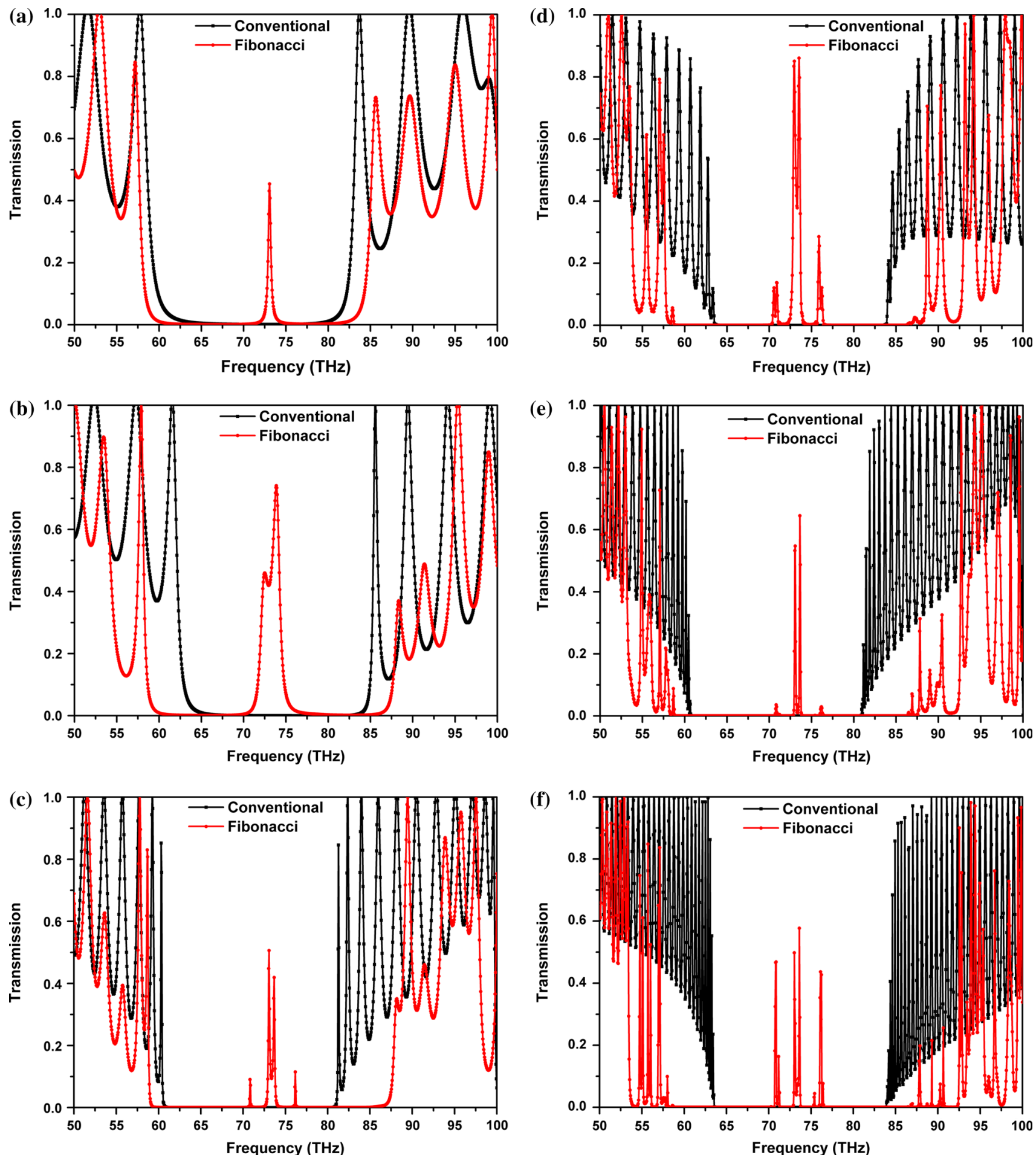

Fig. 3 TE transmittance spectrum of $\mathrm{Sb}_{2} \mathrm{Te}_{3}$ - and $\mathrm{SrTiO}_{3}$-based conventional and Fibonacci photonic crystal structures of a 5th, b 6th, c 7th, d 8 th, e 9 th and $\mathbf{f} 10$ th generations

\subsection{Finite-difference time-domain (FDTD) method and plane wave expansion method (PWE)}

In our calculations, we used OptiFDTD software package [6]. The OptiFDTD software package is based on the finite- difference time-domain (FDTD) method for transmission spectra and the plane wave expansion method (PWE) for photonic band structure.

The photonic band structures of the proposed PCs were calculated by solving the Maxwell equations. The Maxwell 
equation in a transparent, time-invariant, source-free and nonmagnetic medium can be written in the following form:

$$
\nabla \times \frac{1}{\varepsilon(\mathbf{r})} \nabla \times \mathbf{H}(\mathbf{r})=\frac{\omega^{2}}{c^{2}} \mathbf{H}(\mathbf{r})
$$

where $\varepsilon(\mathbf{r})$ is the space-dependent dielectric function, $c$ is the speed of light in vacuum, $\mathbf{H}(\mathbf{r})$ is the magnetic field vector of frequency $\omega$ and time dependence $\mathrm{e}^{j \omega t}$.

This equation is sometimes called the Master Equation and represents an Hermitian eigen-problem, which would not be applicable if the wave equation were derived in terms of the electric field.

$\mathbf{H}(\mathbf{r})=e^{j \mathbf{k r}} \mathbf{h}_{\mathbf{k}}(\mathbf{r})$

where

$\mathbf{h}_{\mathrm{k}}(\mathbf{r})=\mathbf{h}_{\mathbf{k}}(\mathbf{r}+\mathbf{R})$

for all combinations of lattice vectors R. Thus, Maxwell equation is given in operator form:

$(\nabla \times j \mathbf{k}) \times\left[\frac{1}{\varepsilon(\mathrm{r})}(\nabla \times j \mathbf{k})\right] \times \mathbf{h}_{\mathrm{k}}=\frac{\omega^{2}}{c^{2}} \mathbf{h}_{\mathrm{k}}$

By solving this equation for the irreducible Brillouin zone, we can obtain the photonic band structure.

FDTD algorithm is one of the most appropriate calculation tools [7]. For solving Maxwell's equations depending on the time, FDTD algorithm divides the space and time in a regular grid. Perfect matched layers (PMLs) can be used in the determination of the boundary conditions [8]. In general, the thickness of PML layer in overall simulation area is equal to a lattice constant. FDTD solves electric and magnetic fields by rating depending on space and time and deploys rating in different spatial regions by sliding each field component half of a pixel. In our calculations, we have used perfect magnetic conductor (PMC) and anisotropic perfectly matched layer (APML) boundary conditions at $\mathrm{x}$ - and $\mathrm{z}$-directions, respectively.

\section{Results and discussion}

\subsection{Photonic band structure and transmittance}

We calculate the optical properties of $\mathrm{SrTiO}_{3}-\mathrm{Sb}_{2} \mathrm{Te}_{3}$ system up to nth generations $(n=5-10)$. Band structure of $1 \mathrm{D}$ of $\mathrm{SrTiO}_{3}$ - and $\mathrm{Sb}_{2} \mathrm{Te}_{3}$-based CPCs has been calculated in high symmetry directions in the first Brillouin zone (BZ) as shown in Fig. 2. As shown in Fig. 2, there are five photonic band gaps (PBGs) for $\mathrm{Sb}_{2} \mathrm{Te}_{3}$ and $\mathrm{SrTiO}_{3}$ compounds. The widths of the PBGs are (27-43) THz for first,
(68-83) THz for the second, (105-114) THz for the third, (140-149) THz for the fourth and (171-191) THz for the fifth, respectively. When the frequency of the incident electromagnetic wave drops in these PBGs, the electromagnetic wave will be reflected completely by the photonic crystal. As shown in Fig. 2, transmittance is zero in this range of frequencies where the refractive index of the structure is positive and the spectral width of the gaps is invariant with the change in the transmittance (Table 1).

The numerical results of variation of full band gap with changing filling factor from 0.1 to 0.9 are given in Table 1 . Variation of band gap sizes $(\%)$ as a function of filling factor changes between 3.84 and 29.54 for TE1 band. The largest gap sizes are approx. $29 \%$ for $\mathrm{Sb}_{2} \mathrm{Te}_{3}$ and $\mathrm{SrTiO}_{3}$ compounds when filling factor is as high as 0.3 . Then, it decreases when the filling factor continues to increase. On the other hand, when filling factor is 0.3 , the second and fourth gap sizes are going to a minimum. Beside, third and fifth band gap sizes do not change too much according to the filling factor. Then, we have calculated the transmission spectra of conventional and Fibonacci-type photonic crystals with unit cells composed by $\mathrm{Sb}_{2} \mathrm{Te}_{3}$ and $\mathrm{SrTiO}_{3}$ and the same optical thickness for each layer. The spectra are shown in Fig. 3. The position of the minima in the transmission spectrum correlates with the gaps obtained in the calculation. The intensity of the transmission depths is more intense in the case of higher refractive index contrast between the layers. This phenomenon is even more clear for Fibonacci structures (see, Fig. 3a-c).

\section{Conclusions}

The photonic band structures and transmission properties of the $1 \mathrm{D} \mathrm{Sb}_{2} \mathrm{Te}_{3}$ - and $\mathrm{SrTiO}_{3}$-based conventional PCs and Fibonacci PCs were studied. We have investigated transmittance spectra of $\mathrm{SrTiO}_{3}$ - and $\mathrm{Sb}_{2} \mathrm{Te}_{3}$-based both normal PCs and Fibonacci PCs from 5th to 10th generations. The results show that the numbers of pseudoband gaps increase for Fibonacci PCs, when the numbers of the layers increase. The position of the minima in the transmission spectrum correlates with the gaps obtained in the calculation. The intensity of the transmission depths is more intense in the case of higher refractive index contrast between the layers.

Acknowledgements This work is supported by the projects DPTHAMIT, DPT-FOTON, NATO-SET-193 and TUBITAK under Project Nos., 113E331, 109A015, 109E301. One of the authors (Ekmel Ozbay) also acknowledges partial support from the Turkish Academy of Sciences. 


\section{References}

1. S.-V. Gaponenko, Introduction to Nanophotonics (Cambridge University Press, N.Y., 2010)

2. H. Rahimi, A. Namdar, S.-R. Entezar, H. Tajalli, Photonic transmission spectra in one-dimensional Fibonacci multilayer structures containing single-negative metamaterials. Prog. Electromagn. Res. 102, 15-30 (2010)

3. L.-D. Negro, C.-J. Oton, Z. Gaburro, L. Pavesi, P. Johnson, A. Lagendijk, R. Righini, M. Colossi, D.-S. Wiersma, Light transport through the band-edge states of Fibonacci quasicrystals. Phys. Rev. Lett. 90, 055501-055505 (2003)
4. H.H. Landolt, R. Börnstein, Numerical Data and Functional Relationships in Science and Technology-New Series, (Springer, Berlin, 2007), p. 141

5. E.-D. Palik, Handbook of Optical Constants of Solids (Acadamic Press, London, 1998)

6. OptiFDTD 10. http://www.optiwave.com/

7. A. Taflove, S.C. Hagness, Computational Electrodynamics: The Finite-Difference Time-Domain Method (Artech House Publishers, Boston, 2000)

8. J.P. Berenger, A perfectly matched layer for the absorption of electromagnetic waves. J. Comput. Phys. 114, 185-200 (1994) 